\title{
BIOMECHANICAL DETAILS REGARDING THE EFFICIENCY OF START IN SPRINTING EVENTS
}

\author{
Tomina-Dana PETRESCU, Dragoș IONESCU BONDOC
}

\begin{abstract}
Our research is based on a study on the efficiency of athletic start in sprinting events, based on biomechanical analyses of the "key" moments from the start of a sprinter, component of the national athletic team. The study wishes to highlight a number of important details that, under the most favorable conditions, would allow the athlete a better use of the available potential at the start.

The research was based on high-frequency footage recordings of a top runner who ranked third at the National Athletics Indoor Championships.

After analyzing and interpreting the main sequence of the phases of each step after running out of the starting blocks, some technical aspects have been highlighted, which have proved to be restrictive on the performance of the athlete in the competition.

These minor technical errors highlighted in our research may be considered to have made the difference between our subject and the winner of the competition.

We believe that by remedying these small technical deficiencies, through specific analytical exercises, the value of performance of our subject can be substantially improved.

The paper is an analysis of the moments of completion of pulses on the first steps of detachment from the starting block and the moments in which the contact is made with the runway after each phase of flight.

For each of these moments, the impulse angles of the first steps and their growth rate were analyzed, the ground contact angles at landing after each flight phase.
\end{abstract}

Key words: start, contact time, impulse angle, time of detachment.

\section{Introduction}

I consider that the sprinting events are very technical and if you follow the evolution of current performance, we conclude that currently the highest results cannot be obtained unless the athletemastered the correct technique in thes mallest details.

Therefore it is said increasing ly often that at present the difference in major competitions do detail whether the smallest.

\section{Problem Statement}

Looking closely at how to approach the starting positions in the block-start, it is found that there are not two athletes who have the same starting position at the "ready" command.

In most cases, the arguments are subjective, some of them lacking the basis of biomechanical rules, but only on subjective sensations, saying that in that position "I feel best to push in the block-start", others try to mimic the starting positions of some sprinters they saw in various television broadcasts, only because in their evolution they noticed something special to the other athletes, without understanding the degree of efficiency of the movements.

\section{Research Questions/Aims of the research}

Given the complexity of the athletic event, the factors that depend on the results in competitions in the sprint event, among which the most important may be physical training in all aspects, technical training, psychological training, theoretical and tactical training.

It is known that the value of performance in sprint events is particularly influenced by force and, implicitly, power as a form of manifestation.

The question is how and in what report is it with the other factors of training?

\section{Research Methods}

To conduct the study, we have made records of departures from the block start, on command, whose developments we briefly present in this paper.

\section{Findings}

The lower start analysis recorded from the side plan

The arguments are without a scientific basis, some of them being devoid of biomechanical foundation but only on subjective sensations. Representing a series of motor techniques that, when executed correctly, favors the overcoming of inertia - 0 - and the beginning of acceleration as a result of actions of impulse forces including the length of reaction time, the start must allow the muscle groups to engage in the most effective sequence 
Fig. 1. Knee angle of the back leg on the "ready" command

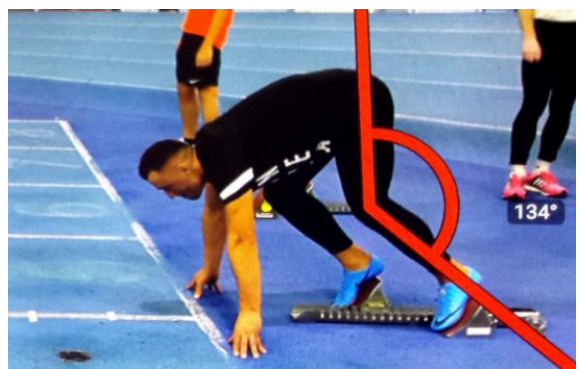

Fig.2. Knee angle of the front leg on the "ready " command

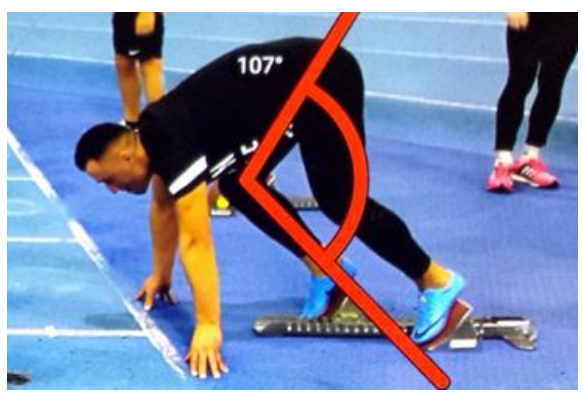

Starting with the "ready" position before the pistol click, (fig 1 and 2) one can notice a decrease of the angles at the knee joint of both legs, which means:

- the increasing the time for achieving the maximum extension of the right foot;

- increasing the amount of energy required to achieve the maximum extension;

- reducing the "maneuvering space" of the legs between the trunk and the ground.

Fig.3. Knee angle of the back of the leg on the driving response

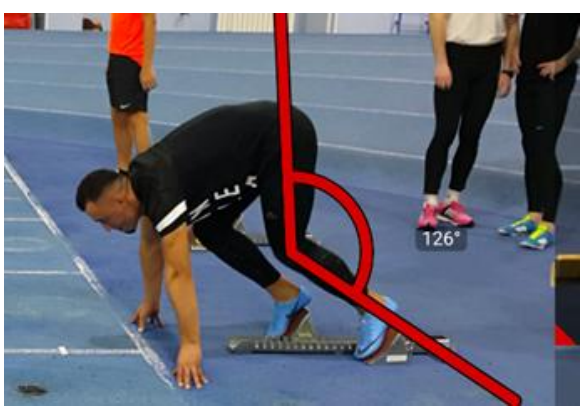

Fig.4. Knee angle of the front legon the driving response

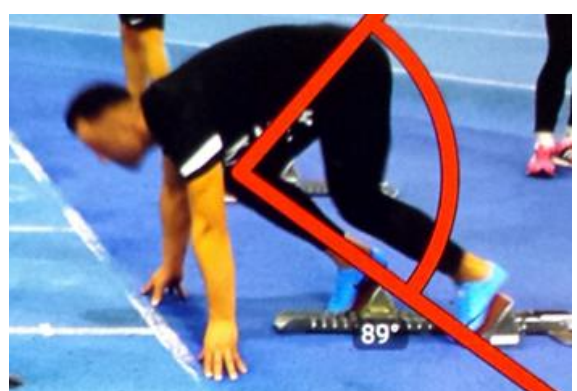

If there is added the space, a few centimeters shorter, between the runway and the athlete's trunk where the running steps is achieved, we can deduce that it could have earned from this sequence a few hundredths of a second. To this is added the difference of time for the maximum extension (fig. 3 and 4) from where we can conclude that the third place he came to the contest, only a few hundredths of a second from the first place, could have been better.

Fig. 5. The moment of completing the impulse of the right foot in the start block

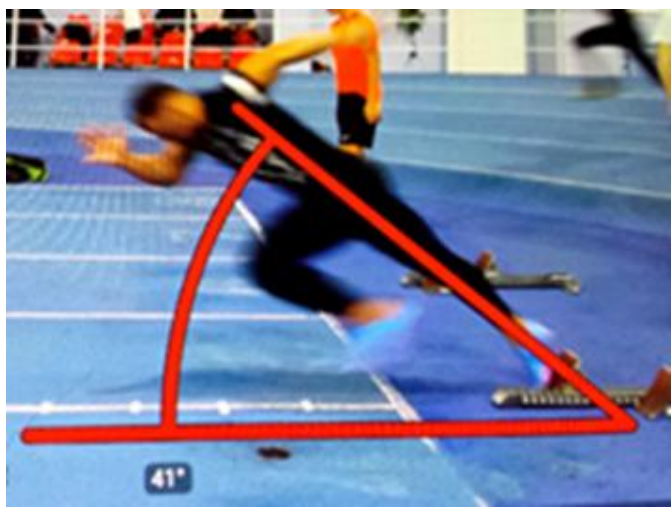

First step. The impulse in the start block. Fig. 5.

Completion of the impulse in the start block is accomplished by a maximum extension of the joints of the extension triple, on the right foot, at an angle of $41^{\circ} \pm 3^{\circ}$, formed by the impulse vector and the runway. It is noticeable that the pendulum segments (left legs and legs) reach the maximum points of the momentum, allowing the athlete to effectively use their inertial forces by cumulating them with the impulse force generated by the right leg fixed on the starting block, closer to the starting line.

The right arm works in a flexion at an angle of $90^{\circ} \pm 3^{\circ}$, while the left arm is almost stretched out from the elbow joint, thus constituting a long pendulum that theoretically has greater inertia but at the same time a faster speed smaller than the right arm. It can be concluded that the inertia of the two arms (long pendulum - short pendulum) works asymmetrically, and diminishes part of the total inertia of the arms.

We can say that there is still a reserve of available biomechanical potential, which can be capitalized. 
Fig.6. Moment of landing in step 2

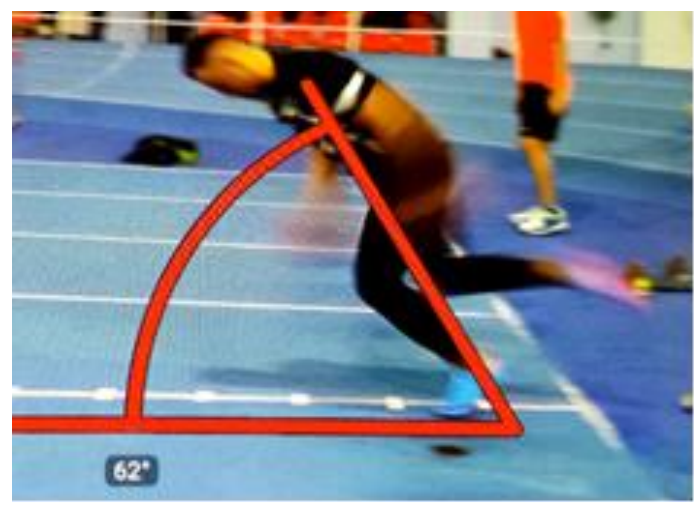

Contact with the soil after the first step (Fig. 6) was made at a distance of about $48 \pm 5 \mathrm{~cm}$ beyond the starting line, on the right foot, at an angle of $62^{\circ} \pm$ $3^{\circ}$, formed by the runway with the vector joining the shoulder axis with the left foot contact point. Ground contact is more "peaked" with a contact time of about $-0.082 \pm 0.033 \mathrm{~s}$, which clearly reveals a locked ankle, insufficiently exploited in this phase. This mode of contact on the ground has the effect of, on the one hand, shortening the distance from the first starting block to the first step contact with the runway, but the negative effect will also be reflected in longer contact times due to the longer duration of damping in the ankle joint (damping-impulse, or otherwise, flexion-extension), which it cannot avoid.

The impulse is performed at an angle of $47^{\circ} \pm$ $3^{\circ}$ with a good coordination of the pendulum segments (arms and the pendulum foot).

\section{Fig. 7. Moment of impulse in step 2}

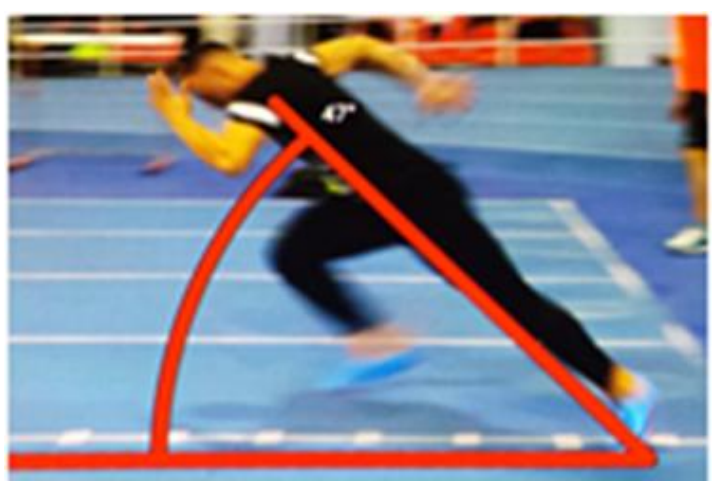

It can be seen the amplitude of the arms flexed from the elbow joint, generating almost identical inerts when they reach the maximum point of the momentum, perfectly synchronized with the maximum point of knee kneeling forward (pendulum). (Fig.7)

This sequence was run over a time frame of $0.104 \pm 0.033 \mathrm{~s}$.

Landing in Step 3 Fig. 8

The flight phase of step two was completed at a distance of about $3.30 \pm 0.05 \mathrm{~m}$, from the starting line on the left foot, with a duration of $0.107 \pm 0.033 \mathrm{~s}$ acting at an angle of about $74^{\circ} \pm 3^{\circ}$, by the vector between the shoulder axis and the runway, after which the support period was $107 \pm 0.033$ s.

Fig.8. Moment of landing in step 3

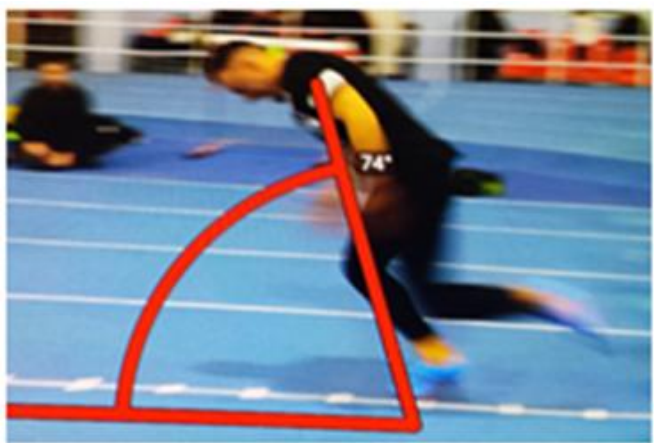

The upward position of the body when landing with the the body mass center projection forward to the ground contact point allows the athlete to maintain and enhance the acceleration of the body while maintaining the optimum balance that allows him to quickly pass into the next impulse phase.

Fig. 9. Completing the Impulse in step 3

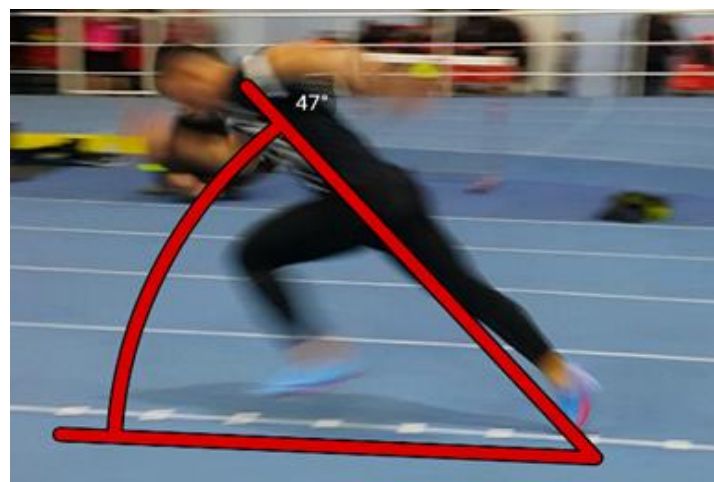

The impulse in step 3. Fig. 9.

The step has evolved as the previous step in similar technical parameters, regarding the impulse angle, determined at the same value of $47^{\circ} \pm 3^{\circ}$, with a good coordination of the pendulum segments (the arms and the pendulum foot). However, the execution speed was lower than the previous step, requiring $0.131 \pm 0.033 \mathrm{~s}$, compared to the previous step time that was $0.104 \pm 0.033 \mathrm{~s}$. This difference can be attributed to the progressive increase in the length of the running step. 
Fig.10. Moment of landing in step 4

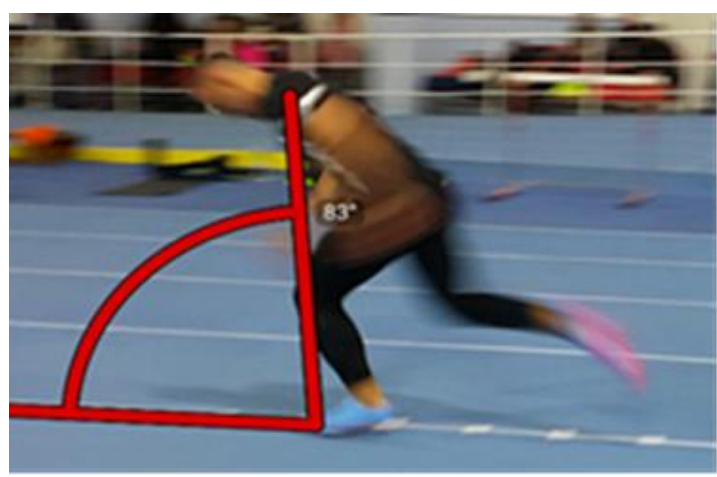

The landing in step 4. Fig. 10.

The moment of contact with the runway at the end of step three was $0.095 \pm 0.033$ s and was made on the left foot at an angle of $83^{\circ} \pm 30$, Increasingly if we take into account the progressive acceleration of the travel speed.

This observation is also confirmed by the increase in the value of the impulse angle in step four, the value of which rose to $49^{\circ} \pm 3^{\circ}$.

The lower start analysis recorded from the front plan

Fig. 11. The moment of completing the impulse of the right leg in the block

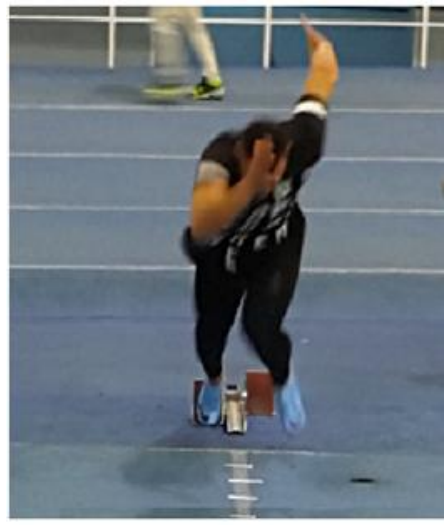

To make a complete analysis of the departure from the start block of our topic, we also made front recoding, shows the moment of impulse completion, indicates a slight leftward leakage of the impulse vector, running and tendency to "screw" the hips around the support axis to avoid the deviations from the running axle, in the next step (fig 11).

The result was a contact with the ground at 30 $\pm 5 \mathrm{~cm}$, left or right (Fig. 12), with respect to the running axle, even if on departure he tries (like Bolt like) a kneeling of the left knee to the right, to the impulse phase in the starting block.

\section{Fig.12. Contact in step1 și 4}

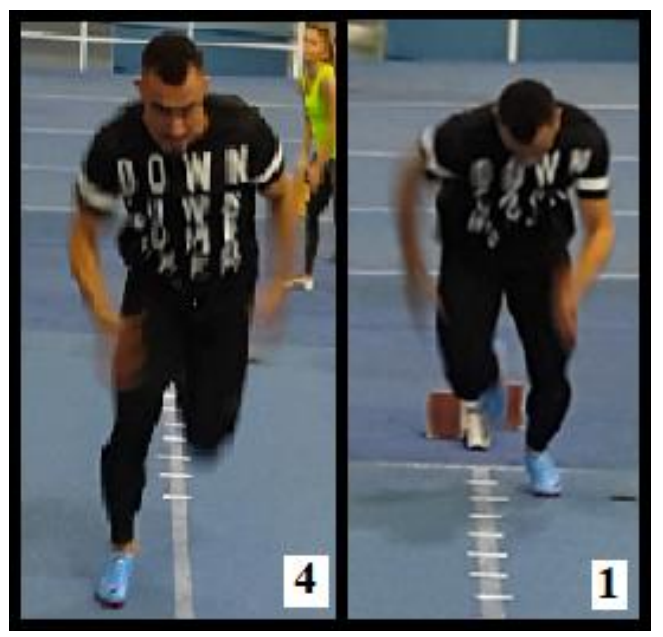

Figure 13. Contact in Step 2 and 3

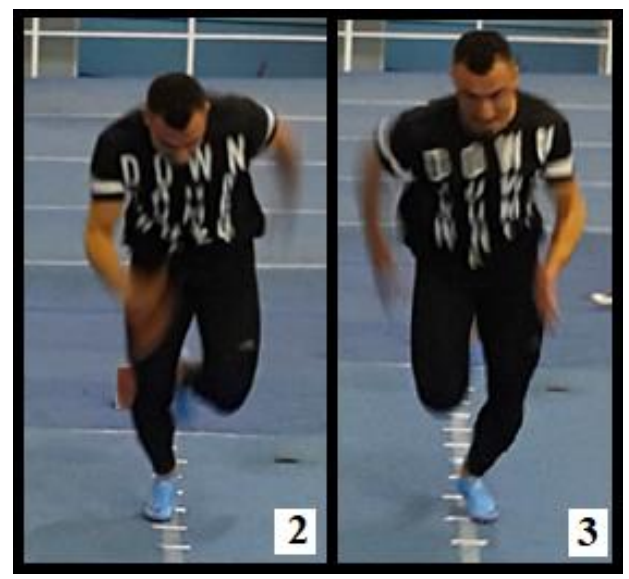

Looking closely at fig. 12 and 13, it can be seen the attempt to return to the central axis of the running direction, also highlighted by the way the pendulum foot is swung to the right. This analysis confirms the evolution of the lateral recordings film technique, where some lateral deviations of the steps can be observed. In the front-mounted recordings we can determine small impulses to deviate from the impulse vectors to the running axis. Fig 13 it is close to the running axis so that in the next step it deviates a lot to the right (fig.12.) the muscular extensions groups to ensure articular stability at the time of receiving the start signal.

\section{Discussions}

In order to solve the problem of the errors resulting from our analysis, it is necessary to work especially for the development of muscle groups to ensure stability in the coxo-femoral joint. Considering that propulsion forces are applied to the runner, and maximum muscle engagement can be considered as an important moment for achieving the best efficacy of the movement. The present exposition will focus, in particular, on the analysis of 
this functional element. The operation of abdominal and lumbar muscles can significantly reduce the deformity of the trunk ligament during the support phase, thus contributing to greater support effectiveness. N. E. Bezodis, in the "Biomechanical Investigations of Sprint and Performance Techniques" - he also cites who did the lower sprint analysis, concluding that in the biomechanical literature the "start" phase was not clearly defined, while some researchers have limited their analysis to the phases and positions in the home block, and others have included one or more steps on the track and of course they are not studies on our theme, but space does not allow us to talk about it all. We believe that all of this, together with the other "small details" that were made, references in this analysis, were reflected in the final result of the $60 \mathrm{~m}$ flat race at the National Athletics Championship on covered ground reserved for seniors, to which our subject ranked third. This observation is also confirmed by the increase in the value of the impulse angle in step four, whose value went up to $83^{\circ}$.

The technical evolution of the support moments analyzed on the basis of the first 4 steps after leaving the block-start, we made out a series of small errors (details).

\section{Conclusion}

This reduction in angles at the knee joint of both legs means:

1. Increase the duration to achieve the maximum extension of the right foot;

2.Increasing the amount of energy required to achieve the maximum extension;

3. Reducing the "maneuvering space" of the legs between the trunk and the ground.

4 . From the moment (0) of the signal reception, until the moment of separation from the support of the arms on the ground, which was determined to be equal to $0.039 \pm 0.033$ seconds, it results that only the correction of this small error can be recovered from the difference that separates him from the 1st place at the $\mathrm{CN}$ of the room.

5. Reducing the maneuvering of the legs in the execution of the treadmill between the trunk and the ground.

6. Making contact on the runway with the ankle joint blocked on the "foot tip" on the first steps is an aspect that our athlete needs to correct.

\section{References}

[1] Bezodis, N., E.; - 2009 Biomechanical investigations of sprint start technique and performance; University of Bath, Author AA, Author BB. Title of article. Abbreviated title of Journal [Internet]. Date of publication YYYY MM [cited YYYY Mon DD]; volume number(issue number):page numbers. Available from: URL
[2] Slow mo Analyse of Best Starting Block Tecnique Asafa Powell;

https://www.youtube.com/watch?v=9IwHjF_CiYc

[3] Speed

Science.

Accelerationhttps://www.youtube.com/watch?v=GZaneGIbi SIanalize

starthttps://www.youtube.com/watch?v=v2_t7R1KcPM 